\title{
The Influence of 24-hr Sleep Deprivation on Psychomotor Vigilance in Young Women and Men
}

This article was published in the following Dove Press journal:

Nature and Science of Sleep

\author{
Marta Ołpińska-Lischka (D) \\ Karolina Kujawa' \\ Josef Alexander Wirth ${ }^{2}$ \\ Katarzyna Z Antosiak- \\ Cyrak $^{3}$ \\ Janusz Maciaszek (D)
}

'Department of Physical Activity and Health Promotion Science, Poznan University of Physical Education, Poznań, Poland; ${ }^{2}$ Institute of Sleep Diagnosis and Therapy, Alfeld, Germany; ${ }^{3}$ Department of Swimming and Water Rescue, Poznan University of Physical Education, Poznań, Poland
Correspondence: Janusz Maciaszek

Poznan University of Physical Education, ul. Królowej Jadwigii 27/39, Poznań 6I-87I,

Poland

$\mathrm{Tel}+48618355214$

Email jmaciaszek@awf.poznan.pl
Background: The number of studies on gender differences in psychomotor performance and sleepiness is small and the results are contradictory. The aim of this study was to assess the changes in psychomotor performance, due to $24 \mathrm{~h}$ of sleep deprivation in young women and men.

Participants: Eighty-nine students (49 women and 40 men) took part in the study. Participants were randomized into two groups: experimental (sleep deprived) and control (non-sleep deprived).

Methods: The research was carried out using computer-based tests of the Vienna Test System (COG, DT, WAFF) and pupillography (F² Dit-For-Duty).

Results: There were no statistically significant effects of the main genders and groups on sleepiness measured by the pupillography. There was no deterioration in the results after deprivation among women and men in the COG test. Changes were noted in the DT and WAFF tests, and their size depended on the test. The number of false responses in psychomotor test was higher in women after sleep deprivation.

Conclusion: One night of sleep deprivation may not have been a negative enough stimulus for young, healthy women and men to reveal gender differences in psychomotor tests. Low sleep levels can lead to low productivity at work and accidents due to reduced vigilance. Insufficient sleep in the long term can lead to poor health, resulting in hypertension, obesity and depression. Keywords: sleep deprivation, psychomotor vigilance, gender difference, pupillography, Vienna Test System

\section{Introduction}

It is estimated that due to the current lifestyle and stress, the sleep duration has decreased by one and a half hours in the last century, ${ }^{1}$ while the hours of morning awakening have not changed. ${ }^{2}$ In developed countries, $5 \%$ of the general population is affected by excessive sleepiness, which occurs almost on a daily basis. ${ }^{3}$

Poor sleep quality often appears among students due to poor sleep hygiene habits. ${ }^{4}$ For many young people, studying provides a degree of independence that they have not experienced before in their family home. Sleep is often irregular when young people start their studies. The reason for this is an intensive lifestyle, which is caused by the students continuing to study and work at the same time. An increasingly common problem among students are the habits associated with the increasing use of media, including in bed, which should only serve to sleep. The use of media in bed helps to make students fall asleep later and reduce the time they spend sleeping. Excessive use of electronic devices in the evening and at night can cause a person to fall asleep later. Light from electronic devices delays the normal 
24-hr rhythm, which increases the excitement of body, which acts as a barrier to starting and maintaining sleep. Lack of sleep reduce vigilance, cognitive performance, motor function and mood, ${ }^{5}$ which may have far-reaching consequences for humans.

Reduced vigilance is a problem in many professions. The effect of reduced vigilance is an increased number of errors and less accuracy. An increased number of errors was found in employees at night shift compared to day shift and the more difficult tasks caused their least accuracy. ${ }^{6}$ In other studies, gender differences were observed, where it was found that women are more accurate than men and have more correct reactions in the test. ${ }^{7}$ Among people with sleep-disordered breathing (SDB), it was observed that men had a greater number of accurate responses than women after sleep deprivation. ${ }^{8}$ Other studies have shown that in visual detection task women were worse than men in detecting correct numerical patterns. ${ }^{9}$ Accuracy is one of the abilities that is used to determine the level of alertness of a surveyed person. ${ }^{10}$ There are very few studies that describe accuracy in psychomotor tests. The overall better accuracy of women than men is pointed out, while the results of studies are not consistent.

Poor quality of sleep or total lack of sleep may result in less effective work. Deterioration of motor coordination is a symptom that occurs after a limited amount of sleep and already one sleepless night causes negative changes in the reaction time. ${ }^{11}$ The reaction time is a very important element of motor coordination, and low vigilance makes it worse, affecting negatively many vital functions. ${ }^{12}$

Sleepiness has a large impact on human functioning and may have a negative impact on many areas of life, primarily affecting human safety. In the medical profession, good coordination of movement is very important, because it requires high vigilance and quick reactions to events. Saadat showed negative changes in the reaction time of anaesthesiologists after $17 \mathrm{hrs}$ of night shift in psychomotor test (PVT). ${ }^{13}$ In a highly developed society where new technologies are used, high concentration is important (eg, to operate machines). Low vigilance not only leads to lower productivity, ${ }^{6}$ but also increases the risk of accidents at work. ${ }^{14}$ Success in sport is often dependent on rapid reaction time, which is one of the key elements to achieve sports championship in many disciplines.

Reaction times vary between men and women and longer reaction times are more common in women than in men. ${ }^{15}$ Tavahomi used DLRT software to evaluate the reaction time by interacting with visual stimuli. The test consisted of pressing a button when squares appeared. There were no differences in reaction time between women and men. ${ }^{16}$ With the help of Deluxe Multi-Choice Reaction Time Apparatus (Lafayette Instrument Company), tests were conducted on students who also did not reveal significant statistical differences in reaction time. ${ }^{17}$

The number of studies on gender differences and lack of sleep and its effects is small and the results are contradictory. However, authors of existing studies on experimental sleep deprivation rarely consider gender as a potential variable. This is probably because researchers are more likely to generalize their research samples in order to make them similar to those of the population. There is a small number of studies that determine the level of reaction time and response accuracy in psychomotor tests for both genders after sleep deprivation. Therefore, the aim of this study is to investigate the differences after $24 \mathrm{hrs}$ of sleep deprivation in young women and men.

The following research hypotheses have been formulated:

1. The level of sleepiness will increase in all subjects after 24-hr sleep deprivation.

2. $24 \mathrm{hrs}$ sleep deprivation causes a higher number of incorrect reactions in selected coordination tests (Vienna Test System) in women than in men.

3. $24 \mathrm{hrs}$ sleep deprivation results in a longer reaction time in women than in men.

\section{Methods}

\section{Ethics Statement}

This project was reviewed and approved by the Ethics Committee of the Poznan University of Medical Sciences in Poland, approval number 989/17. All participants were included in the study after signing written informed consent in accordance with the Declaration of Helsinki.

\section{Participants}

The average age for the whole experimental group was 22 \pm 1.70 years; control group $23 \pm 1.32$ years. Body mass index in the control group was $23.39 \pm 2.61$ years, while in the experimental group $23.12 \pm 2.72$ years. Young students of physical education not reporting health problems were included in the study group. Respondents were randomly divided into experimental and control groups. During recruitment, all participants were personally screened and they received explanations regarding the 
inclusion and exclusion criteria from the study. We went to physical education students participating in bachelor and master studies. Information about the research was announced before and after the lectures. Before qualifying students for the study, surveys and interviews were conducted to include or exclude students from the study. Then, the students signed the informed consent to participate in the study. Criteria for inclusion in the experiment: bachelor and master degree students, resignation from alcoholic beverages and beverages containing caffeine on the day of test, last meal up to 8 p.m. not exceeding 350 kcal and consent to participate in tests. Exclusion criteria: age under 18, age over 30. Also subjects with sleep disorders, psychiatric disorders (which were clinically diagnosed in the past), acute conditions or who were taking sleeping pills were excluded from participation.

\section{Procedure}

All tests were carried out on working days. The research was conducted at the University of Physical Education in Poznań, as it is the most suitable place from the point of view of a research project (computers, laboratory equipment). The control group had slept at home but arrived in the laboratory for testing. All students were informed about maintaining their usual amount of sleep before starting the experiment. Except for the questionnaire method, the amount of sleep was not checked the night before the examination with any tools.

The initial stage of the study consisted of conducting a zero test. The functioning of each psychomotor test was explained to students before the experiment. Each student had been trained once. The training was aimed at ensuring that students understand the tests and conduct them properly.

We decided to use 24-hr sleep deprivation because research suggests that people who are physically active may have better results in reaction time. ${ }^{18,19}$ In connection with these reports, it was considered that partial deprivation might be too small a stimulus for the differences to occur. $24 \mathrm{hrs}$ prior to test, students were provided with actigraphs to measure their daily physical activity. The last meal was consumed until 8 p.m. The calorific value of the last meal was calculated on the basis of the caloric content of the products. The meal prepared by researchers was provided to all students. During the test, activation forms were planned for student participants, such as board games, playing cards, puzzles. The measurements were conducted in the morning $(6.00-8.00)$ on the first day (session 1) and at the end of $24 \mathrm{hrs}$ of sleep deprivation on the next day (session 2).

\section{Vienna Test System}

The research was carried out using computer-based tests of the Vienna Test System, which is used in psychomotor measurements and meets the criteria of test quality (accuracy, reliability and objectivity) (www.schuhfried.at/wts/neuro/).

The following standardized tests were used in studies: COG - Cognitrone (S7), which measures the attention and concentration levels. The test compares geometric figures to indicate whether the displayed figure is identical to the top row figure. The test person determines this with a green button on the control panel. Tested variables: sum of correct and incorrect reactions, time of correct and incorrect reactions. DT - Determination Test (S4) to evaluate stress tolerance and complex reactions. The respondent's task is to react to stimuli that follow one another. The task is to distinguish one of the two sounds and one of the five colors. At the same time, the respondent must press the same button on the control panel that is displayed on the screen. In this test, the pedals for the left and right leg are used. Test variables: number of correct and incorrect reactions, number of missed reactions, number of stimuli, total stimuli. The WAFF Focused attention test is used to assess the focus of attention. The respondent focuses on two geometric figures. The task is to react to stimulus when two figures are highlighted. Otherwise, the respondent ignores the stimuli. Test variables: number of omitted and incorrect reactions, reaction time.

\section{Pupillography}

The sleepiness level was also evaluated using a pupillography ( $F^{2} \mathrm{D}$ Fit-For-Duty), which consists of a video camera with infrared light (light emitting diodes), filming the respondent's pupil in the dark for 11 mins. $^{20}$ The measurement enables an objective evaluation of sleepiness. The final result is expressed as a PUI value (pupillary unrest index). The PUI is classified at three levels: values up to $6.64 \mathrm{~mm} / \mathrm{min}$ mean "normal" sleepiness. A range of values from 6.65 to $9.8 \mathrm{~mm} / \mathrm{min}$ indicates "elevated" sleepiness, while PUI above $9.8 \mathrm{~mm} / \mathrm{min}$ is characterized as "pathological" sleepiness.

\section{Epworth Sleepiness Scale}

In the first period of this study, sleepiness was also assessed in all participants by means of a diagnostic survey method using the Epworth Sleepiness Scale (ESS). ${ }^{21}$ 
The Epworth Sleepiness Scale (ESS) determines the respondent's sleepiness for the last 4 weeks. This tool consists of eight statements. The final result enables to determine the degree of sleepiness during the day (0-5 points - "low normal sleepiness", 6-10 points - "higher normal sleepiness", 11-12 points - "mild excessive sleepiness", 13-15 points - "moderate excessive sleepiness", 16-24 points - "severe excessive sleepiness").

\section{Statistical Analysis}

A two-factor analysis of ANOVA variance was used to calculate the effects of sleep deprivation on the parameters of Vienna System tests, determining the main effects of interactions. Distribution normality was verified by the Shapiro-Wolf test. Pearson's Chi 2 test was used to assess the relationship between individual category variables. The general characteristics of results were presented as the median $(\mathrm{M}) \pm$ standard deviation (SD) and average $\left(M_{e}\right)$. Statistical significance was determined at the level of $\mathrm{p}<0.05$.

\section{Results}

In total, 89 students (49 women and 40 men) took part in the study. PUI $>9.8 \mathrm{~mm} / \mathrm{min}$ indicates a very high risk of falling asleep in accordance with the standards. In the experimental group, after sleep deprivation, this indicator was slightly lower in women $\left(M_{e}=9.83\right)$ than in men $\left(M_{e}=10.84\right)$, but still indicated a very high risk of falling asleep. In none of the groups was a result below $\mathrm{PUI}<6.6 \mathrm{~mm} / \mathrm{min}$, which would indicate normal values.

There were no statistically significant effects of the main genders and groups on sleepiness measured by the pupillography. There were no interactive effects between the studied effects. Measurement of sleepiness in the first term in both control group genders showed very high sleepiness (women $M_{e}=13.22$; men $M_{e}=13.46$ ). The analysis showed that the sleepiness of the examined women and men from the control group in the first term was very high. Sleepiness was slightly higher in women than in men. PUI in the second term was significantly higher in women than in men (women $M_{e}=17.15$; men $M_{e}=12.53$ ).

Epworth Sleepiness Scale was used to assess the degree of daily sleepiness. ESS scores, which are in the range of 6-10 points, indicate higher normal daytime sleepiness and are considered normal. This was observed in $50 \%$ of all men. Higher levels of normal sleepiness were also found in women in general (51.02\%). Values in the range of 13-15 points indicate moderate excessive sleepiness during the day. No significant differences were found between men and women (total women 14.29\%; total men $15 \%$ ). Only $4 \%$ of women had severe sleepiness.

A two-factor analysis of ANOVA variance in scheme 2 (gender) $\times 2$ (control group vs experimental group) was carried out, where the result of particular test of the Vienna System was a variable.

With regard to the number of mistakes in WAFF test, statistical analysis showed that there was no main effect of gender $(p=0.26)$. The analysis did not reveal the effect of interaction between the groups according to gender $(\mathrm{F}(1.78)$ $=0.11 ; \mathrm{p}=0.73$ ). This means that between the two measurements (after sleep and sleepless nights) there were no statistically significant differences between the control and experimental groups depending on gender. Women in the experimental group were characterized by a higher number of mistakes $\left(M_{e}=2.32 ; 1 \pm 6.3\right)$ than men $\left(M_{e}=1.62 ; 1 \pm 2.65\right)$ in the measurement after sleepless nights (Figure 1).

Further analysis in the reaction time measurement by the same test also carried out in scheme 2 (gender: women, men) $\times 2$ (control and experimental group) showed that the main effect of gender and group was statistically insignificant. However, there was an interactive effect of the group. This means that the reaction time increased in the experimental group after sleep deprivation compared to the time after sleep at night $(\mathrm{p}=0.04)$.

There was also an interaction of gender and group depending on the measurement $(\mathrm{F}(1.78)=6.40 ; \mathrm{p}=0.01)$. A significant effect of interaction means that in different measurements the respondents had different reaction times depending on the gender and group they belonged to. The obtained result indicates the relation between gender and groups depending on the measurement. The post hoc test showed a difference with the tendency to significance $(p=0.06)$ in the group of women in the control group between the first study and second study. In addition, it was found that in case of women from the experimental group, the reaction time after sleep deprivation significantly increased $\left(M_{e}=408.77 \mathrm{~ms}\right)$ in comparison to men from the same group $\left(M_{e}=362.87 \mathrm{~ms}\right.$ ) (Figure 2).

In DT test, no significant statistical main effects were observed for both genders and groups. However, the statistical interaction of the group was significantly observed. Differences between the control group and experimental group were found, where it was noted that the experimental group after sleep deprivation had a slightly longer reaction time $(\mathrm{M}=0.68 \mathrm{~s})$ than the control group $(M=0.63 \mathrm{~s})(\mathrm{p}=0)$. There was no significant statistical interaction between the group and gender in the measurements $(\mathrm{F}(1.98)=0.10$, 


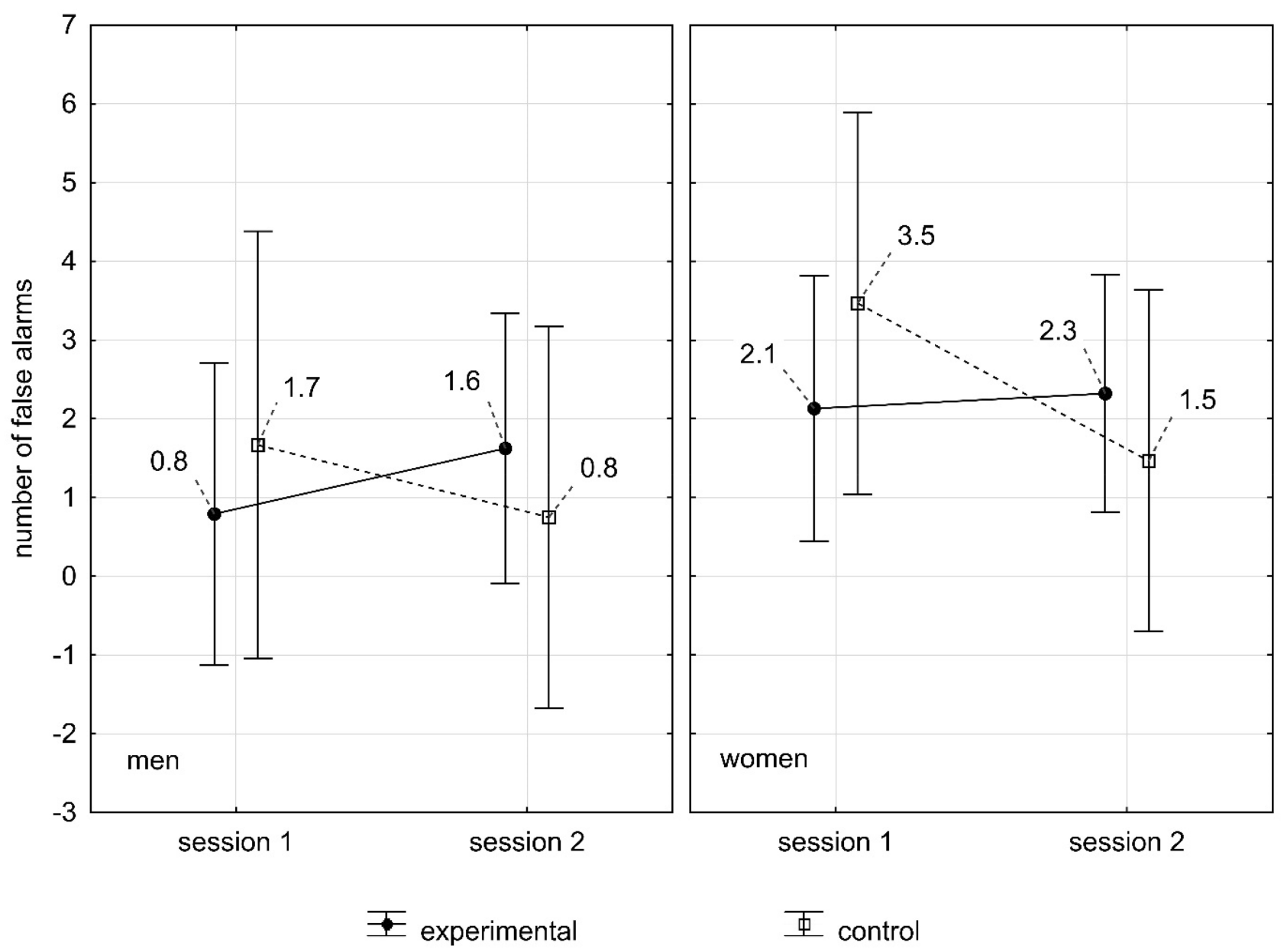

Figure I The influence of sleep deprivation on the number of mistakes made in WAFF test in women and men.

$\mathrm{p}=0.75)$. After sleep deprivation, the results of women $(M=$ $0.67 \mathrm{~s})$ and men in the experimental group $(M=0.68 \mathrm{~s})$ were similar. In the second measurement of comparative group, the results were similar (women $M=0.66 \mathrm{~s}$; men $M=0.66 \mathrm{~s}$ ) (Figure 3).

\section{Discussion}

Reaction time, response accuracy and sleepiness in young healthy women and men were measured. We used tools that are considered reliable. Their advantage is also ease of use and their objectivity. Two major findings emerged from this study. The first important conclusion was the lack of gender differences in the measurement of student sleepiness measured by the pupillography. Another important observation was to show that lack of sleep worsens reaction time in some test in women. Psychomotor tests also showed lower female accuracy in responses.

Pupillography is a fast and objective method that can be carried out in almost all conditions. The experimental group had lower results after sleep deprivation than after a sleepy night $\left(1^{\text {st }}\right.$ period $)$. Nevertheless, the sleepiness of respondents was at a similar very high level. Moreover, there were no gender differences. Wilhelm showed that PUI in the pupillography test also did not correlate with gender. Students from the experimental group before sleep deprivation achieved similar results (5.58 \pm 3.04$)$ as after sleep deprivation (5.93 $\pm 3.26) .{ }^{22}$ Physicians before sleep deprivation (5.05 \pm 3.19$)$ had a higher PUI score than after sleep deprivation (4.47 $\pm 2.14) .{ }^{23}$ Probably the aspect that may have affected relatively small changes between women and men, and between sleep deprivation and slept night ( $1^{\text {st }}$ period), is the students' adaptation to reduced sleep time due to their lifestyle. During the examination period, students often have worse sleep hygiene. Most of them have shorter sleeping times due to increased learning for better learning outcomes. Some of them are also active at work (often at night), which makes them more tolerant to fatigue. A factor that further increases fatigue tolerance is physical activity. ${ }^{24}$ 


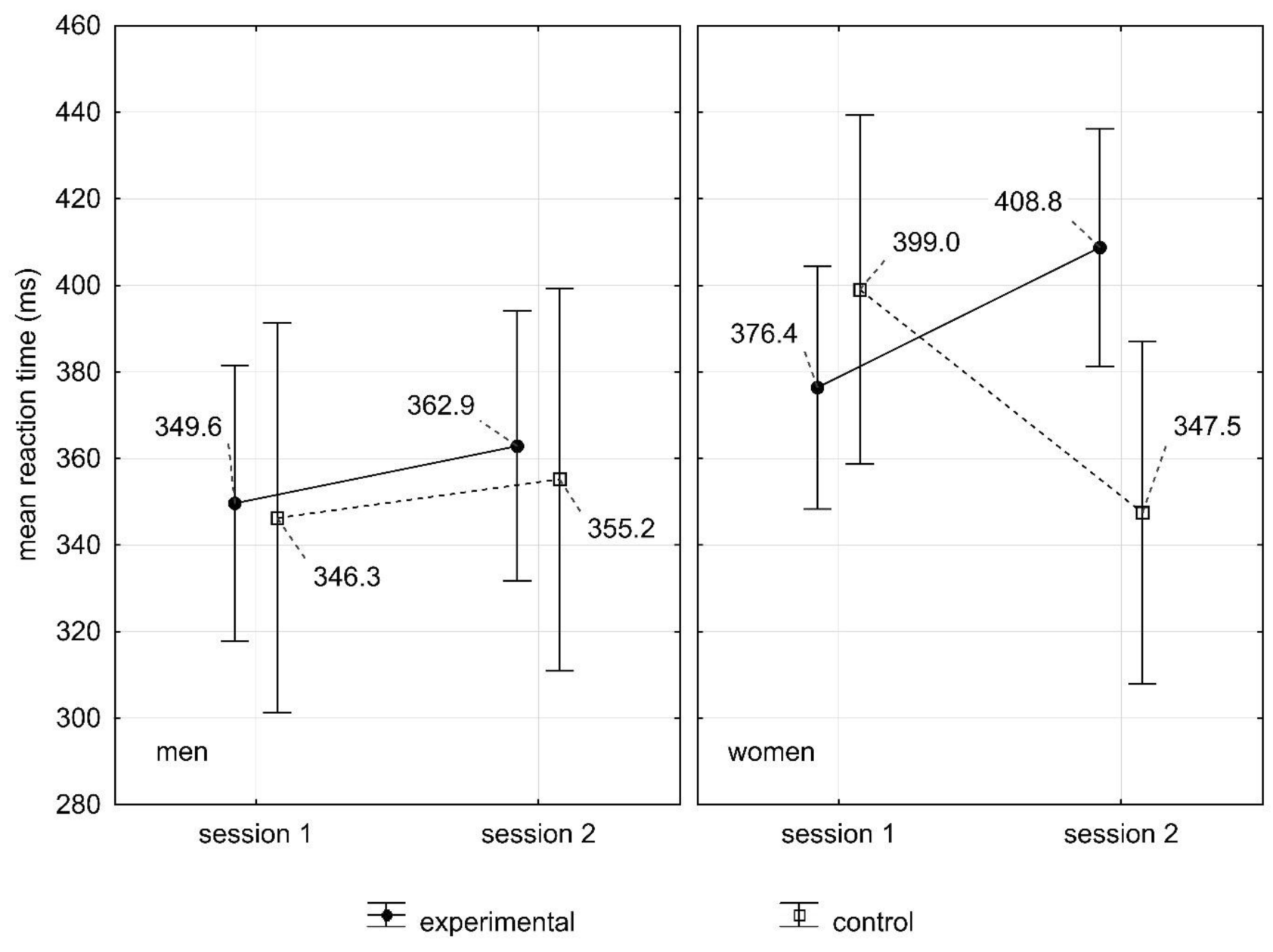

Figure 2 The influence of sleep deprivation on the reaction time made in WAFF test in women and men.

Further results to evaluate sleepiness using the ESS scale showed that subjectively felt sleepiness in the last 4 weeks was higher in women $\left(M_{e}=8.85\right)$ than in men $\left(M_{e}\right.$ $=7.34$ ), and their sleepiness was classified as higher than normal. The ESS scale is used to assess the habitual level of sleepiness in a healthy population and clinical populations. This scale is one of the most commonly used measures of sleepiness. ${ }^{21}$ The repeatability of the results is defined as moderately sufficient. ${ }^{25}$ The ESS sleepiness measurement was carried out to determine the overall habitual degree of sleepiness in the longer period preceding these studies. However, the main part of the experiment was the assessment of the actual alert state using a pupilograph. The parameters of the ESS scale and the pupilograph cannot be compared due to other parameters.

Pupillography is considered as a reliable measure of sleepiness. ${ }^{26}$ Surprisingly, sleepiness in the experimental group after sleep deprivation was lower than after a normal night's sleep, but it was still at a very high level. Usually, PUI values after sleep deprivation are higher. Unchanged or lower sleepiness results after the pupillography test may occur in professional groups that perform shift work. These groups often adapt to the nature of work. ${ }^{23}$ It is difficult to explain the lower sleepiness results after sleep deprivation in students. Perhaps the sleepiness of students decreased due to the participation in an experiment and measurements, which were very attractive and interesting for the majority of respondents. The participation in tests could have a stimulating effect, which probably resulted in an increase in the adrenaline level. Increased levels of this hormone increase vigilance for some time. ${ }^{27}$ Although the PUI index in the experimental group was lower after sleep deprivation, the tendency to fall asleep was still very high according to the accepted standards. Higher PUI values after sleep deprivation were observed in men. The level of PUI after sleep deprivation was lower than after sleep at night (1st period). Moreover, these results show that many people are not aware of the real level of their sleepiness and assess it better. 

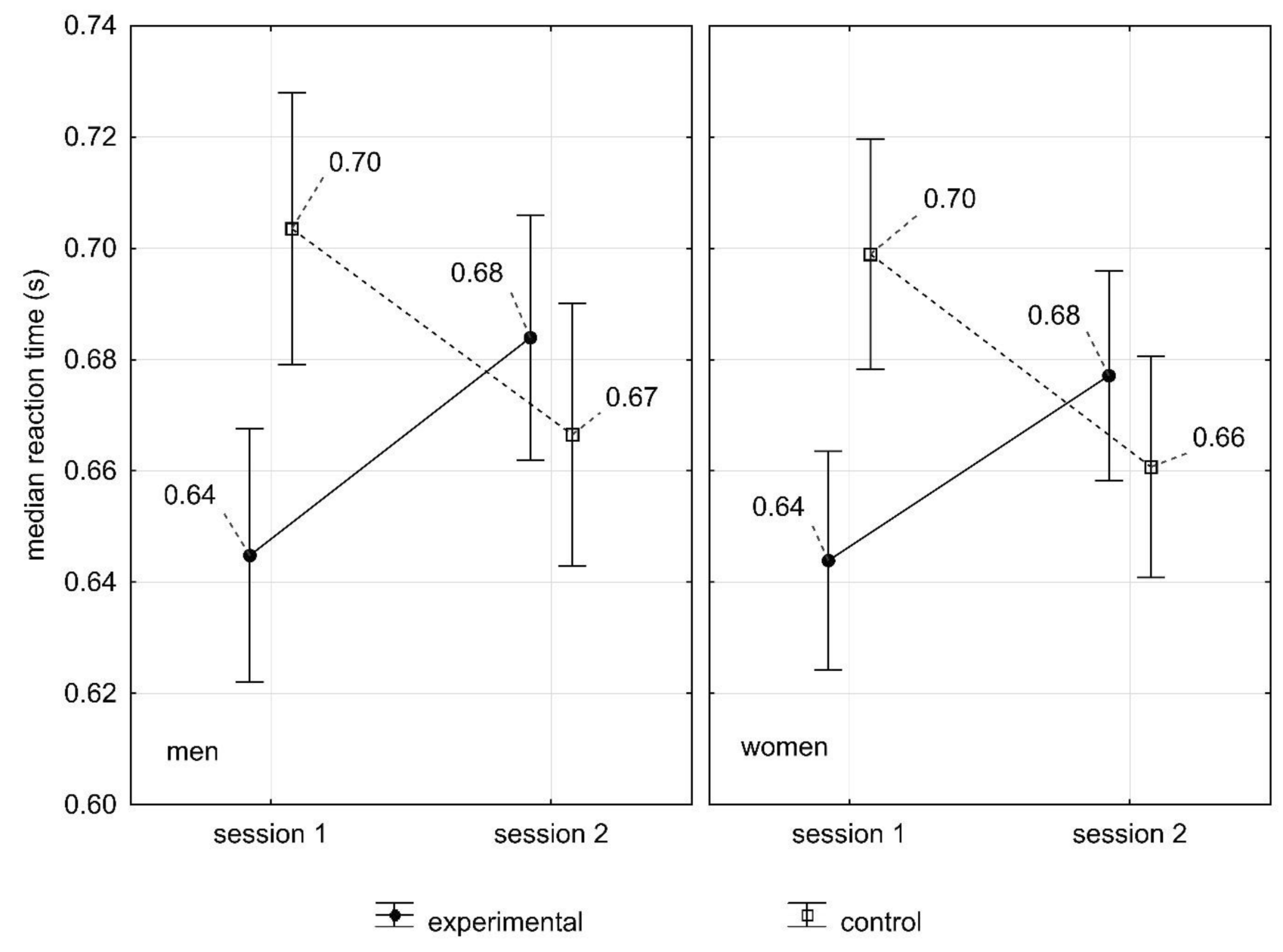

Figure 3 The influence of sleep deprivation on the reaction time made in DT test in women and men.

After sleep deprivation, the results of visual reaction time in WAFF test deteriorated in both women and men $(p<0.05)$. The study by Karia aimed at evaluating the reaction time to visual stimuli in students proved that women had a longer reaction time than men. ${ }^{28} \mathrm{~A}$ longer reaction time of young, agile female students before and after sleep deprivation was also found in our experiment. Although auditory and visual stimuli reach the cerebral cortex in both women and men at the same time, muscle contraction occurs faster in men. Therefore, women have a longer reaction time than men. ${ }^{29}$

The results of DT reaction time after sleep deprivation deteriorated slightly $(\mathrm{p}<0.05)$, and in comparison to sleepy nights $\left(1^{\text {st }}\right.$ period $)$, the difference was small. In this sample, no gender influence was found $(F(1.98)=0.10$, $\mathrm{p}=0.75$ ). Gender differences are more noticeable in tests affecting visual than auditory stimuli. ${ }^{30}$ The DT test affects visual and auditory reactions. Such a small difference between women and men could be due to the type of test used. DT consists of tasks that require rapid reactions, and the participant reacts to the signals by using his feet, both hands, and additionally concentrating on the sound signals. Physical education students usually have a high level of physical activity. ${ }^{31}$ Other studies found that also physically active medical students had better results compared to the physically inactive group. ${ }^{32}$ Therefore, regular physical activity improves the reaction time. Measurement of reaction time in the experimental group of women and men slightly deteriorated and was longer in men.

The hypothesis that the reaction time will be prolonged in women and men after sleep deprivation is justified. Significant differences after sleep deprivation between women and men were observed in the WAFF test - in women the reaction time was longer. The range of changes after sleep deprivation in DT test between women and men, regardless of the study group and dates, was less noticeable. This means that one night of sleep deprivation resulted in a longer reaction time in DT test, but it was 
men who had a slightly longer reaction time than women. It was observed that the nature of selected tests may have been the cause of deterioration in reaction time. The form of DT test is very dynamic, which requires constant attention. However, the WAFF test is invariable, with a repetitive task, which is tedious for the person performing it. Due to the monotonous nature, the respondent may have reduced vigilance. Unvarying and long tests may reduce the level of vigilance in sleepiness. In the conducted COG measurement no gender differences were found in the experimental group. Using the same test, Kramm also did not notice any differences between women and men. ${ }^{33}$ Sleep deprivation did not affect results in the experimental group of women and men, because the results did not change. Women had less correct reactions $\left(M_{e}=46\right)$ than men $\left(M_{e}=48\right)$, which worsened after sleep deprivation (women with $M_{e}=0.05$; men with $M_{e}=0.03$ ) compared to the first measurement $\left(1^{\text {st }}\right.$ period). The correctness of reaction did not show any differences between the control and experimental groups. In such tests, it is often stated that women have more correct reactions, but slower reaction times. ${ }^{7}$ This hypothesis cannot be confirmed because the women in the experimental group had less correct reactions than the men regardless of the date. In this task, sleep deprivation did not cause any deterioration in the concentration of women and men. The sum of incorrect reactions worsened only in women after sleep deprivation (men $M_{e}=47.33$; women $M_{e}=47.51$ ) compared to the test after sleep at night (men $M_{e}=47.95$; women $\left.M_{e}=47.35\right)$. Results were at a similar level in both terms. No gender impact was noted ( $p>0.05)$. It is difficult to explain the lower accuracy in responses of women compared to men. Both tests are used to measure attention. In general, women have better attention, but men have higher motor speed. ${ }^{34}$ The lower accuracy in responses of women in the WAFF test results can be explained by better motor skills of men. This is confirmed by the results of the reaction time parameter in this test, where men had shorter reaction times (Figure 2). In addition, the attention process may be correlated with the phase of the menstrual cycle, ${ }^{35}$ which could potentially affect our test results. It is worth conducting further tests to determine these relationships.

The experiment also had some limitations. The first limitation was that no longer sleep deprivation was used, which would have allowed more information to be obtained and stronger changes to be noticed. In subsequent studies among students of physical education, when investigating the influence of sleep deprivation on psychophysical abilities, it is worth applying more difficult tests. Due to the obtained results, it is also worthwhile to introduce tests that are long.

In addition, it is worth considering the assessment of the subjects' chronotype, as a correlation between chronotype and sleep time for cognitive abilities may be found. ${ }^{36}$ Chronotype differences determine a person's performance at a given time of day. This means that wounded types are characterized by greater performance in the morning. A different pattern is observed in the evening type, ${ }^{37}$ which may affect the results. Unfortunately, this factor was not investigated in the experiment, and therefore the determination of the chronotype in subsequent studies seems necessary and may give interesting results and show further potential dependencies.

\section{Conclusions}

In summary, a very high level of sleepiness measured by the pupilograph in men and women was found. These findings suggest excessive sleepiness for students that may have health consequences. The effects of a lack of sleep can be short-lived and can manifest as stress, depression, anxiety, decreased performance, and cognitive deficits. Poor hygiene of sleep may cause long-term effects of lack of sleep, which are associated with the occurrence of hypertension or the appearance of metabolic syndrome. ${ }^{3,8}$ The right amount of sleep is a factor that is very important for maintaining good health.

There was no deterioration in the results after deprivation among women and men in the COG test. Changes were noted in the DT and WAFF tests, and their size depended on the test. A clear deterioration was observed in the WAFF test during the response. Differences in reaction time after sleepless night between women and men in the DT test were insignificant. We also found lower accuracy in women's responses in psychomotor tests after sleep deprivation.

The analysis of results shows that sleep deprivation has led to worse results in some tests. However, one night of sleep deprivation may not have been a negative enough stimulus for young, healthy women and men to reveal gender differences. In subsequent studies, it is, therefore, worthwhile to include other age groups or students from other universities in tests in order to observe gender changes and to apply longer sleep deprivation. More complex research needs to be carried out in order to generalize our findings. 


\section{Acknowledgment}

The authors would like to thank all the participants who took part in this study.

\section{Funding}

This research did not receive any specific grant from funding agencies in the public, commercial, or not-forprofit sectors.

\section{Disclosure}

No potential conflict of interest was reported by the authors.

\section{References}

1. Meerlo P, Sgoifo A, Suchecki D, Restricted and disrupted sleep: effects on autonomic function, neuroendocrine stress systems and stress responsivity. Sleep Med Rev. 2008;12:197-210. doi:10.1016/j. smrv.2007.07.007

2. Carskadon M, Acebo C, Seifer R. Extended nights, sleep loss and recovery sleep in adolescents. Arch Ital Biol. 2001;139:301-312.

3. Ohayon M. Epidemiology of excessive daytime sleepiness. Sleep Med Clin. 2006;1(1):9-16. doi:10.1016/j.jsmc.2005.11.004

4. Meyer-Szary J, Jakitowicz M, Sieczkowski M, Kasperczyk J, Jakitowicz J. The quality of sleep in college students Tricity. Sen 2008;8(1):15-21.

5. Durmer JS, Dinges DF. Neurocognitive consequences of sleep deprivation. Semin Neurol. 2005;25(1):117-129. doi:10.1055/s-2005867080

6. Santhi N, Horowitz TS, Duffy JF, Czeisler CA. Acute sleep deprivation and circadian misalignment associated with transition onto the first night of work impairs visual selective attention. PLoS One. 2007;8(11):e1233. doi:10.1371/journal.pone.0001233

7. Barral J, Debu B. Aiming in adults: sex and laterality effects Laterality: assymmetries of body. Brain Cog. 2004;9(3):299-312.

8. Kim H, Dinges DF, Young T. Sleep-disordered breathing and psychomotor vigilance in a community-based sample. Sleep. 2007;30 (10):1309-1316. doi:10.1093/sleep/30.10.1309

9. Mäntylä T. Gender differences in multitasking reflect spatial ability. Psychol Sci. 2013;24(4):514-520. doi:10.1177/0956797612459660

10. Flehmig HC, Steinborn M, Langner R, Scholz A, Westhoff K. Assessing intraindividual variability in sustained attention: reliability, relation to speed and accuracy, and practice effects. Psychol Sci. 2007;49(2):132-149.

11. Taheri M, Arabameri E. The effect of sleep deprivation on choice reaction time and anaerobic power of college student athletes. Asian $J$ Sports Med. 2012;3(1):15-20. doi:10.5812/asjsm

12. Mathis J, Hess CW. Sleepiness and vigilance tests. Swiss Med Wkly. 2009;139(15-16):214-219. doi:smw-12498

13. Saadat H, Bissonnette B, Tumin D, et al. Effects of partial sleep deprivation on reaction time in anesthesiologists. Paediatr Anaesth. 2017;27(4):358-362. doi:10.1111/pan.13035

14. Lockley SW, Barger LK, Ayas NT, Rothschild JM, Czeisler CA, Landrigan CP. Harvard work hours, health and safety group. Effects of health care provider work hours and sleep deprivation on safety and performance. Jt Comm J Qual Patient Saf. 2007;33(11):7-18. doi:10.1016/S1553-7250(07)33109-7

15. Blatter K, Graw P, Münch M, Knoblauch V, Wirz-Justice A, Cajochen C. Gender and age differences in psychomotor vigilance performance under differential sleep pressure conditions. Behav Brain Res. 2006;168(2):312-317. doi:10.1016/j.bbr.2005.11.018
16. Tavahomi M, Shanbehzadeh S, Abdollahi I. Comparing the effect of fatigue on choice reaction time of healthy men and women. Phys Treat. 2017;7(1):29-34.

17. Teleb AA, Al Awamleh AA. Gender differences in cognitive abilities. Curr Res Psychol. 2012;3(1):33-39. doi:10.3844/crpsp.2012.33.39

18. Jain A, Bansal R, Kumar A, Singh DK. A comparative study of visual and auditory reaction times on the basis of gender and physical activity levels of medical first year students. Int J Appl Basic Med Res. 2015;5(2):124-127. doi:10.4103/2229-516X.157168

19. Nakamoto H, Mori S. Sport-specific decision-making in a Go/NoGo reaction task: difference among nonathletes and baseball and basketball players. Percept Mot Skills. 2008;106(1):163-170. doi:10.2466/ pms.106.1.163-170

20. Lüdtke $H$, Wilhelm B, Adler $M$, Schaeffel $F$, Wilhelm $H$. Mathematical procedures in data recording and processing of pupillary fatigue waves. Vision Res. 1998;38(19):2889-2896. doi:10.1016/ S0042-6989(98)00081-9

21. Johns MW. A new method for measuring daytime sleepiness: the epworth sleepiness scale. Sleep. 1991;14(6):540-545. doi:10.1093/sleep/14.6.540

22. Wilhelm B, Giedke H, Luedtke H, Bittner E, Hofmann A, Wilhelm H. Daytime variations in central nervous system activation measured by a pupillographic sleepiness test. J Sleep Res. 2001;10 (1):1-7. doi:10.1046/j.1365-2869.2001.00239.x

23. Kupietz E. Fatigue After Duty - Subjective Perception or Objective Fact? [dissertation]. Philipps-Universität Marburg; 2015.

24. Härmä M. Ageing, physical fitness and shift work tolerance. Appl Ergon. 1996;27(1):25-29. doi:10.1016/0003-6870(95)00046-1

25. Terry JL, Heil DP. Test-retest reliability of the epworth sleepiness scale in healthy adults. Int J Exerc Sci. 2016;8(4):64.

26. Morad Y, Lemberg H, Yofe N, Dagan Y. Pupillography as an objective indicator of fatigue. Curr Eye Res. 2008;21(1):535-542. doi:10.1076/0271-3683(200007)2111-ZFT535

27. Howard SK. Sleep deprivation and physician performance: why should I care?. Bayl Univ Med Cent. 2005;18(2):108-112.

28. Karia RM, Ghuntla TP, Mehta HB, Gokhale PA, Shah CJ. Effect of gender difference on visual reaction time: a study on medical students of Bhavnagar region. IOSR J Pharm. 2012;2(3):452-454.

29. Silverman IW. Sex differences in simple visual reaction time: a historical meta-analysis. Sex Roles. 2006;54(1-2):57-69. doi:10.1007/s11199-006-8869-6

30. Solanki J, Joshi N, Shah C, Mehta HB, Gokhle PA. A study of correlation between auditory and visual reaction time in healthy adults. Int J Med Sci Public Health. 2012;2(2):8-10.

31. Baj-Korpak J, Korpak F, Szepeluk A, Sudoł G. Factors conditioning the motives and obstacles to physical activity of students of physical education. Soc Dissertations. 2016;10(4):60-72.

32. Kramm T. Standardization Study of a Attention-Test Battery (Vigilance, Signal-Detection and Cognitrone of the Vienna Test System of Schuhfried [dissertation]. Ruhr- Universität Bochum; 2000.

33. Roalf DR, Gur RE, Ruparel K, et al. Within-individual variability in neurocognitive performance: age and sex-related differences in children and youths from ages 8 to 21. Neuropsychology. 2014;28 (4):506-518. doi:10.1037/neu0000067

34. Upadhayay N, Guragain S. Comparison of cognitive functions between male and female medical students: a pilot study. J Clin Diagn Res. 2014;8(6):BC12-BC15. doi:10.7860/JCDR/2014/7490.4449

35. Vetter C, Juda M, Roenneberg T. The influence of internal time, time awake, and sleep duration on cognitive performance in shiftworkers. Chronobiol Int. 2012;29(8):1127-1138. doi:10.3109/07420528.2012. 707999

36. Roenneberg T, Wirz-Justice A, Merrow M. Life between clocks: daily temporal patterns of human chronotypes. J Biol Rhythms $J$. 2003;18(1):80-90. doi:10.1177/0748730402239679

37. Medic G, Wille M, Hemels ME. Short- and long-term health consequences of sleep disruption. Nat Sci Sleep. 2017;9:151-161. doi:10.2147/NSS.S134864 


\section{Publish your work in this journal}

Nature and Science of Sleep is an international, peer-reviewed, open access journal covering all aspects of sleep science and sleep medicine, including the neurophysiology and functions of sleep, the genetics of sleep, sleep and society, biological rhythms, dreaming, sleep disorders and therapy, and strategies to optimize healthy sleep.
The manuscript management system is completely online and includes a very quick and fair peer-review system, which is all easy to use. Visit http://www.dovepress.com/testimonials.php to read real quotes from published authors. 